\title{
PLATINOSSOMOSE EM FELINO DOMÉSTICO NO MUNICÍPIO DE PELOTAS, RS, BRASIL
}

\author{
Alexsander Ferraz ${ }^{1}$ \\ Camila Moura de Lima ${ }^{2}$ \\ Eugênia Tavares Barwaldt ${ }^{3}$ \\ Eduarda Santos Bierhals ${ }^{4}$ \\ Bruno Cabral Chagas ${ }^{1}$ \\ Jéssica Paola Salame ${ }^{4}$ \\ Andreza Bernardi da Silva ${ }^{3}$ \\ Leandro Quintana Nizoli ${ }^{5}$ \\ Márcia de Oliveira Nobre ${ }^{5}$
}

\begin{abstract}
RESUMO
Platynosomum spp. é um trematódeo, da família Dicrocoeliidae, que tem como hospedeiros definitivos, os felídeos, mas também pode parasitar primatas e aves silvestres. Animais que possuem o hábito de se alimentar de répteis ou anfíbios, são mais susceptíveis. Acomete principalmente o fígado e os ductos biliares, mas pode ser eventualmente encontrado também no intestino delgado, ductos pancreáticos, pulmões e outros tecidos. A fisiopatologia inclui quadro de colangite crônica, podendo se estender e acometer o parênquima hepático e culminar com colangiohepatite, fibrose biliar, cirrose e obstrução biliar. Os sinais clínicos variam conforme a gravidade do caso e duração da infecção. As manifestações clínicas incluem anorexia, letargia, perda de peso, hepatomegalia, distensão abdominal e vômitos, podendo ocorrer, ainda, icterícia e alteração de consistência das fezes. Os gatos adultos, não domiciliados ou domiciliados com acesso à rua que possuem hábitos de caça, são mais predispostos. O diagnóstico definitivo pode ser feito pela pesquisa parasitológica e presença de ovos em análise coproparasitológica, pela identificação de ovos na bile e mais frequentemente por histopatologia hepática. O tratamento consiste no uso de anti-helmínticos, sendo que o mais eficaz é o praziquantel. Além disso, medidas preventivas devem ser adotadas, como evitar que os gatos tenham acesso aos hospedeiros intermediários. O presente relato, consiste no diagnóstico coproparasitológico de platinossomose em dois felinos domésticos, no município de Pelotas, RS, Brasil.
\end{abstract}

Palavras chave: felinos, colangiohepatite, trematódeo hepatobiliar, lagartixa.

\section{PLATINOSOMOSIS IN FELINE DOMESTIC IN THE MUNICIPALITY OF PELOTAS, RS, BRAZIL}

\begin{abstract}
Platynosomum spp. is a trematode, from the family Dicrocoeliidae, which has felids as its definitive host, but can also parasit primates and wild birds. Animals that have a habit of feeding on reptiles or amphibians, are more susceptible. It mainly affects the liver and bile ducts, but it can eventually be found also in the small intestine, pancreatic ducts, lungs and

\footnotetext{
${ }^{1}$ Doutorando na Universidade Federal de Pelotas. Correspondência: xanderferraz@yahoo.com.br

${ }^{2}$ Mestrando na Universidade Federal de Pelotas. camila.moura.lima@ hotmail.com

${ }^{3}$ Graduanda em Medicina Veterinária. Universidade Federal de Pelotas. tbeugenia@gmail.com

${ }^{4}$ Residente Clínica Médica Animais de Companhia, Universidade Federal de Pelotas. dudabierhals@hotmail.com

${ }^{5}$ Professor Adjunto Universidade Federal de Pelotas. marciaonobre@ gmail.com
} 
other tissues. Pathophysiology includes chronic cholangitis, which may extend and affect the liver parenchyma and culminate in cholangiohepatitis, biliary fibrosis, cirrhosis and biliary obstruction. Clinical signs vary according to the severity of the case and the duration of the infection. Clinical manifestations include anorexia, lethargy, weight loss, hepatomegaly, bloating and vomiting, and jaundice and alteration of stool consistency may also occur. Adult cats, homeless or homeless with access to the street who have hunting habits, are more predisposed. The definitive diagnosis can be made through parasitological research and presence of eggs in coproparasitological analysis, by identifying eggs in the bile and more often through hepatic histopathology. Treatment consists of the use of anthelmintics, the most effective of which is praziquantel. In addition, preventive measures must be adopted, such as preventing cats from having access to intermediate hosts. The present report consists of the coproparasitological diagnosis of platinosomosis in two domestic cats, in the municipality of Pelotas, RS, Brazil

Keywords: felines, cholangiohepatitis, hepatobiliary trematode, lizard.

\section{PLATINOSOMOSIS EN FELINOS DOMÉSTICOS EN EL MUNICIPIO DE PELOTAS, RS, BRASIL}

\section{RESUMEN}

Platynosomum spp. es un trematodo, de la familia Dicrocoeliidae, que tiene a los felinos como hospedadores definitivos, pero también puede parasitar primates y aves silvestres. Los animales que tienen la costumbre de alimentarse de reptiles o anfibios, son más susceptibles. Afecta principalmente al hígado y los conductos biliares, pero eventualmente también se puede encontrar en el intestino delgado, los conductos pancreáticos, los pulmones y otros tejidos. La fisiopatología incluye la colangitis crónica, que puede extenderse y afectar el parénquima hepático y culminar en colangiohepatitis, fibrosis biliar, cirrosis y obstrucción biliar. Los signos clínicos varían según la gravedad del caso y la duración de la infección. Las manifestaciones clínicas incluyen anorexia, letargo, pérdida de peso, hepatomegalia, distensión abdominal y vómitos, e ictericia y alteración de la consistencia de las heces. Los gatos adultos, sin hogar o sin hogar con acceso a la calle que tienen hábitos de caza, están más predispuestos. El diagnóstico definitivo se puede realizar mediante la investigación parasitológica y la presencia de huevos en el análisis coproparasitológico, mediante la identificación de los huevos en la bilis y más a menudo mediante la histopatología hepática. El tratamiento consiste en el uso de antihelmínticos, el más eficaz de los cuales es el praziquantel. Además, se deben adoptar medidas preventivas, como evitar que los gatos tengan acceso a huéspedes intermediarios. El presente informe consiste en el diagnóstico coproparasitológico de platinosomosa en dos gatos domésticos, en el municipio de Pelotas, RS, Brasil.

Palabras clave: felinos, colangiohepatitis, trematodo hepatobiliar, lagartija.

\section{INTRODUÇÃO}

Platynosomum spp. (família Dicrocoelidae) é o parasito hepático mais comum em felinos domésticos (Felis catus) e geralmente está localizado nos ductos biliares e vesícula biliar (1). Entretanto, pode acometer intestino delgado, ductos pancreáticos, pulmões e outros tecidos, sendo comumente associado à colangite e colangiohepatite (2). 
No Brasil, já foi relatada ocorrência do parasito no Ceará (3), Santa Catarina (4), Paraíba (5), Bahia (6), Rio Grande do Norte (7), Minas Gerais (8). No Rio Grande do sul, foi relatada em Porto Alegre (9).

O ciclo do Platynosomum spp. exige a presença de dois a três hospedeiros intermediários, sendo os isópodos e moluscos terrestres, os primeiros e lagartixas e sapos, os segundos hospedeiros intermediários. A principal via de infecção nos gatos ocorre pela ingestão de lagartixas, contendo as metacercárias (forma infectante) $(10,11)$.

Os achados laboratoriais incluem elevados níveis de enzimas hepáticas (FA, AST, ALT e GGT) e hiperbilirrubinemia (1). O diagnóstico é realizado pela história do paciente, junto com o exame físico e exames complementares, e o diagnóstico definitivo pode ser feito por exame coproparasitológico, pela detecção de ovos operculados nas fezes. Dessa forma, o presente trabalho teve como objetivo, relatar os casos de dois felinos diagnosticados com Platinossomose no município de Pelotas, RS, Brasil.

\section{RELATO DE CASO}

Os dois pacientes atendidos eram fêmeas felinas, sendo que a paciente do caso 1 era da raça siamesa, 10 anos, apresentando como sinais clínicos: inapetência, febre $\left(39,5{ }^{\circ} \mathrm{C}\right)$, taquicardia (196 bpm), vômito, anorexia, emagrecimento gradual, icterícia e hepatomegalia na palpação abdominal. Enquanto a paciente do caso 2 era sem raça definida, 14 anos, com histórico de apatia e hiporexia. Os tutores buscaram atendimento para check-up em função da idade avançada e da apatia e hiporexia recente. No exame clínico, a única alteração encontrada foi a visualização de mucosa oral levemente ictérica.

Após avaliação clínica de ambos os casos, foi solicitado exames de ultrassonografia abdominal, hemograma e perfil bioquímico pela pesquisa da alanina aminotransferase (ALT), aspartato transaminase (AST), glutamiltransferase (GGT), fosfatase alcalina (FA), albumina, creatinina e ureia. Após os resultados destas análises, realizou-se também avaliação coproparasitológica pela técnica de sedimentação espontânea

\section{DISCUSSÃO}

Os resultados dos exames do primeiro caso, revelaram na ultrassonografia abdominal, hepatomegalia, com lobos hepáticos com contornos arredondados, parênquima hiperecogênico, indicando lipidose hepática. Além disso, constatou-se espessamento de parede da vesícula biliar, com presença significativa de lama biliar, levando a possível diagnóstico de colangite. A paciente do caso 2 não apresentou alterações neste exame de imagem. Tanto os achados clínicos quanto os do ultrassom da paciente 1 são os encontrados em casos de platinossomose, como: icterícia, anorexia, letargia e perda de peso, os quais são comuns às doenças hepáticas obstrutivas (12), É importante considerar que sintomatologia pode ser inespecífica, como inapetência, letargia, anorexia e perda de peso. Sendo os sinais mais evidentes, vômitos, diarreia, anemia, hepatomegalia, ascite e icterícia (2). A gravidade dos sinais clínicos é proporcional ao número de parasitos adultos no hospedeiro e a duração deste parasitismo (13).

Na platinossomose é comum observar-se, na ultrassonografia abdominal, dilatação de ductos biliares, distensão e espessamento da parede da vesícula biliar, como tortuosidade e dilatação dos ductos biliares e distensão da vesícula biliar além de hepatomegalia $(1,12)$. Os gatos domésticos diagnosticados com platinossomose, geralmente possuem acima de dois anos de idade, histórico de ingerir lagartixas e costumam ter acesso à rua ou quintais (14).

No exame hematológico da paciente do caso 1, foram observadas alterações na leucometria, caracterizado por leucocitose $(20.100 \mu \mathrm{L})$ e neutrofilia $(17.889 \mu \mathrm{L})$. O plasma 
estava ictérico com presença de agregações plaquetárias. As enzimas hepáticas AST e ALT estavam acima do limite fisiológico, com 94,6 UI/L e 107, 4 UI/L, respectivamente. Porém, o maior aumento deu-se nos valores da fosfatase alcalina (FA), cujo valor de referência utilizado é de 25-93 UI/L, e o exame desta paciente mostrou valor de 1273,4UI/L.

Já a paciente do caso 2 apresentou no exame hematológico, diminuição de hemácias, hematócrito e hemoglobina. Também foi observado nível elevado de GGT e FA, com respectivamente $11 \mathrm{UI} / \mathrm{L}$ e $268 \mathrm{UI} / \mathrm{L}$. Ambos os felinos apresentaram aumento da FA, sendo que na do primeiro caso observou-se um aumento bastante acentuado. FA é a enzima que deve ser dado maior atenção no quadro da platinossomose. Em estágios iniciais da doença, geralmente não manifesta considerável alteração (15), porém, em estágios mais avançados e inflamação evidente no colédoco e vesícula biliar, alterações extremas de valores são notadas (16).

O diagnóstico primário de ambos os pacientes foi colangio-hepatite felina. Pelo fato dos sinais clínicos serem compatíveis com platinossomose, solicitou-se também, avaliação coproparasitológica, onde foi possível detectar, pela técnica de sedimentação espontânea, a presença de ovos compatíveis com os de Platynosomum spp. (Fig. 1), pois estes são marrons, de casca espessa, operculados e simétricos, medindo em média de 34 a $50 \mu \mathrm{m}$ por 20 a $35 \mu \mathrm{m}$ (17).

O diagnóstico definitivo pode ser confirmado pela pesquisa parasitológica e presença de ovos em análise coproparasitológica, presumindo que os parasitas não obstruíram por completo o ducto biliar, pela identificação de ovos na bile e também por histopatologia hepática $(18,19)$. Desta forma, o exame fecal pode ser dificultado pela pequena quantidade de ovos que passam para as fezes diariamente, como também pela presença de ovos imaturos e maduros, que possuem morfologia diferente, além do pequeno tamanho dos ovos (3).

A técnica coproparasitológica de eleição para identificação do Platynosomum spp., é a de sedimentação espontânea, sendo mais eficiente, quando comparadas com as técnicas de flutuação de Willis-Mollay e Faust (20). Isto deve-se ao fato dos ovos de Platynosomum spp. serem pesados, portanto, melhor diagnosticado por métodos de sedimentação (21). Sobral et al. (22), estudando a prevalência da infecção por Platynosomum illiciens em gatos domésticos de Araguaína, TO, determinaram que a sedimentação espontânea foi o teste que demonstrou maior sensibilidade e especificidade no diagnóstico deste parasito.

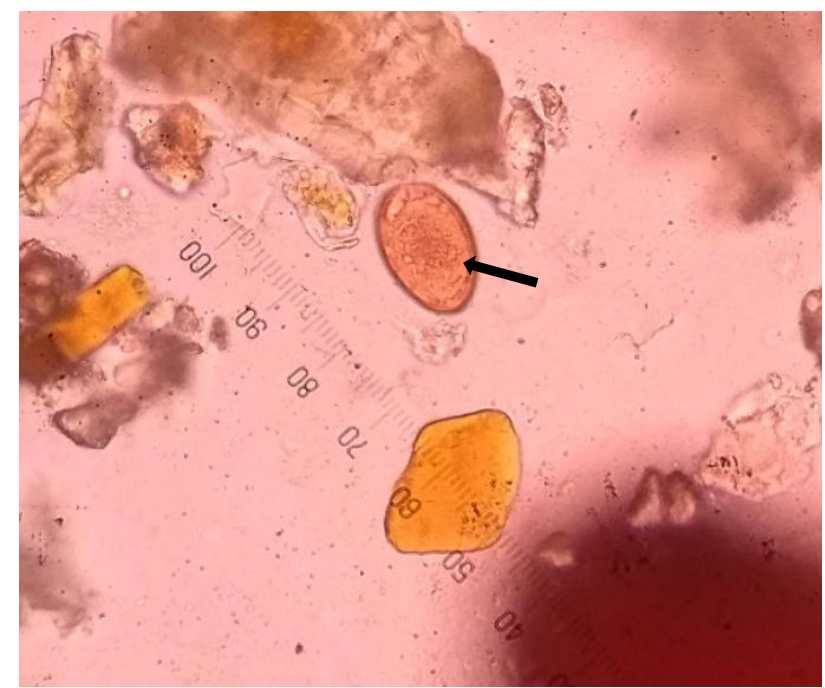

Figura 1. Ovo de Platynosomum spp. (seta) em fezes de felino doméstico, após realização da técnica de sedimentação espontânea, observado em microscopia óptica, aumento de 400x. Fonte: Autor, 2019 
A terapia para a paciente do primeiro caso, consistiu em tratamento sintomático com fluidoterapia com ringer lactato ( $1 \mathrm{gota} / \mathrm{s})$, ondasetrona $(0,2 \mathrm{~mL}, \mathrm{IV}, 8 \mathrm{em} 8$ horas), e para analgesia utilizou-se tramadol $(0,05 \mathrm{~mL}, \mathrm{IV}, 12 \mathrm{em} 12$ horas). Além disso, como o paciente não estava alimentando-se, optou-se pela colocação de sonda esofágica, possibilitando a alimentação forçada, realizada de 4 em 4 horas. Após o diagnóstico de Platinossomose, foi instituído terapia com praziquantel $(50 \mathrm{mg} / \mathrm{kg})$, via oral, a cada 24 horas por 5 dias. Porém, mesmo com o tratamento, devido as lesões hepáticas e biliares, o animal veio a óbito.

Para o paciente do segundo caso, foi prescrita terapia antiparasitária com praziquantel, na dose de $50 \mathrm{mg} / \mathrm{kg}$ via oral a cada 24 horas durante 5 dias e ácido ursodesoxicólico, um medicamento indicado para o tratamento de doenças hepato-biliares e colestáticas crônicas, na dose de $20 \mathrm{mg} / \mathrm{kg}$ via oral a cada 24 horas durante 30 dias. Após o tratamento observou-se melhora do quadro clínico, onde constatou-se que a paciente havia normalizado o apetite, além das mucosas não apresentarem mais alteração de coloração. No hemograma observou-se melhora significativa dos valores de hemácias, hematócrito e hemoglobina e no exame coproparasitológico não foi mais observado ovos de Platynosomum spp.

O tratamento com praziquantel na dose de $50 \mathrm{mg} / \mathrm{kg}$ por via oral, uma vez ao dia por 5 dias foi eficaz, sendo considerado o tratamento de eleição (23). A eficácia do tratamento, depende do grau de lesão no fígado, ducto biliar e vesícula biliar (24). É o agente antihelmíntico mais eficaz contra o P. factosum, podendo ser administrado por via subcutânea ou via oral (25). O praziquantel aumenta a permeabilidade de membrana aos íons cálcio, interferindo na captação da glicose por cestódeos e trematódeos, causando morte por paralisia flácida (26). O tratamento de suporte deve ser realizado concomitantemente, dando enfoque ao suporte nutricional com dieta de alta proteína, que deve ser realizado de forma precoce e intensiva. Além disso, deve ser preconizado fluidoterapia de suporte nos estágios iniciais do tratamento (12). Em animais que apresentarem anorexia por um longo período é recomendado a administração de um estimulante de apetite ou colocação de sonda nasoesofágica (27).

\section{CONCLUSÕES}

A platinossomose deve ser investigada em casos de felinos com aumento da atividade sérica das enzimas hepáticas e colestásicas, sendo o exame coproparasitológico uma das formas de diagnosticá-la. Por meio desta análise foi possível realizar o diagnóstico desta parasitose nos pacientes deste relato, possibilitando a prescrição do tratamento adequado para os casos. Se o mesmo for realizado de forma tardia, a evolução da doença pode levar o animal a óbito, fato ocorrido em um dos pacientes deste relato.

\section{REFERÊNCIAS}

1. Daniel AGT, Diaz RF, Camignatto LO, Kage NK, Pellegrino A, Cogliati B. Polycystic liver associated with Platynosomum fastosum infection in a cat. Braz J Vet Pathol [Internet]. 2012 [cited 2020 May 28];5(3):137-41. Available from: https://bjvp.org.br/wpcontent/uploads/2015/07/DOWNLOAD-FULL-ARTICLE-29-

20881_2012_11_30_54_12.pdf

2. Lima GS, Dabus DMM, Trentin TC, Neves MF. Platynosomum factosum. Rev Cient Eletronica Med Vet [Internet]. 2008 [cited 2020 May 28];6(11):1-6. Available from: http://www.faef.revista.inf.br/imagens_arquivos/arquivos_destaque/qfVQ8Y1AaRhrE Tw_2013-6-13-15-31-11.pdf 
3. Sousa Filho RP, Sampaio KO, Holanda MSB, Vasconcelos MC, Morais GB, Viana DA, et al. Primeiro relato de infecção natural pelo Platynosomum spp. em gato doméstico no município de Fortaleza, Ceará, Brasil. Arq Cienc Vet Zool UNIPAR [Internet]. 2015 [cited 2020 May 28];18(1):59-63. Available from: https://www.revistas.unipar.br/index.php/veterinaria/article/view/5370

4. Moraes AV, Frondana L, Fischer AL, Sturion T, Kitamura EA, Claus MP, et al. Relato do primeiro diagnóstico parasitológico de Platynosomum Looss (1907) em felino no estado de Santa Catarina. In: Mostra Nacional de Iniciação Cientifica e Tecnológica Interdisciplinar; 2015; Araquari. Araquari: Instituto Federal Catarinense; 2015.

5. Andrade RLSF, Dantas AFM, Pimentel LA, Galiza GJN, Carvalho FKL, Costa VMM, et al. Platynosomum fastosum - induced cholangiocarcinomas in cats. Vet Parasitol [Internet]. 2012 [cited 2020 May 28];190(1-2):277-80. Available from: https://www.sciencedirect.com/science/article/abs/pii/S0304401712002130?via\%3Dih $\mathrm{ub}$

6. Sampaio MAS, Berlim CM, Angelim AJGL, Gondim LFP, Almeida MAO. Infecção natural pelo Platynosomum Looss 1907, em gato no município de Salvador, Bahia. Rev Bras Saude Prod Anim [Internet]. 2006 [cited 2020 May 28];7(1):1-6. Available from:https://repositorio.ufba.br/ri/bitstream/ri/1883/1/642-2485-2-PB.pdf

7. Ahid SMM, Filgueira KD, Suassuna ACD, Feijó FMC, Alves ND. Ocorrência de Platynosomum fastosum (Trematoda: Dicrocoeliidae) em gato doméstico (Felis catus) em Mossoró-RN. Nosso Clinico. 2005;8(47):66-70.

8. Mundim TCD, Oliveira SD Jr, Rodrigues DC, Cury MC. Frequência de helmintos em gatos de Uberlândia, Minas Gerais. Arq Bras Med Vet Zootec [Internet]. 2004 [cited 2020 May 28];56(4):562-3. Available from: https://www.scielo.br/pdf/abmvz/v56n4/21998.pdf

9. Michaelsen R, Silveira E, Marques SMT, Pimentel MC, Costa FVA. Platynosomum concinnum (Trematoda: Dicrocoeliidae) em gato doméstico da cidade de Porto Alegre, Rio Grande do Sul, Brasil. Vet Foco [Internet]. 2012 [cited 2020 May 28];10(1):53-60. Available from: http://www.periodicos.ulbra.br/index.php/veterinaria/article/view/1167

10. Basu AK, Charles RA. A review of cat liver fluke Platynosomum fastosum Kossack, 1910 (Trematoda: Dicrocoeliidae). Vet Parasitol [Internet]. 2014 [cited 2020 May 28];200(1-2):1-7. Available from: https://www.sciencedirect.com/science/article/abs/pii/S0304401713006821

11. Pinto HA, Mati VL, De Melo AL. New insights into the life cycle of Platynosomum (Trematoda: Dicrocoeliidae). Parasitol Res [Internet]. 2014 [cited 2020 May 28];113(7):2701-7. Available from: https://link.springer.com/article/10.1007/s00436014-3926-5

12. Watson PJ. Doenças hepatobiliares no gato. In: Nelson RW, Couto CG. Medicina interna de pequenos animais. 5a ed. Rio de Janeiro: Elsevier; 2015. Cap.37, p.536-58.

Ferraz A, Lima CM, Barwaldt ET, Bierhals ES, Chagas BC, et al. Platinossomose em felino doméstico no município de Pelotas, RS, Brasil. Vet. e Zootec. 2021; v28: 001-008. 
13. Salomão MS, Souza Dantas LM, Mendes de Almeida F, Branco AS, Bastos OPM, Sterman F, et al. Ultrasonography in hepatobiliary evaluation of domestic cats (Felis catus, L., 1758) infected by Platynosomum Loss, 1907. Int J Appl Res Vet Med [Internet]. 2005 [cited 2020 Jun 2];3(3):271-9. Available from: https://www.jarvm.com/articles/Vol3Iss3/SALAMAO.pdf

14. Rodriguez-Vivas RI, Williams JJ, Quijano-Novelo AG, Bolio GM, Torres-Acosta JF. Prevalence, abundance and risk factors of liver fluke (Platynosomum concinnum) infection in cats in Mexico. Vet Rec [Internet]. 2004 [cited 2020 Jun 2];154(22):693-4. Available from: https://veterinaryrecord.bmj.com/content/154/22/693

15. Ramos DGS, Santos ARGLO, Freita LC, Braga IA, Silva EP, Soares LMC, et al. Feline platynosomiasis: analysis of the association of infection levels with pathological and biochemical findings. Rev Bras Parasitol Vet [Internet]. 2017 [cited 2020 Jun 4];26(1):54-9. Available from: https://www.scielo.br/pdf/rbpv/v26n1/1984-2961-rbpvS1984-29612017009.pdf

16. Linton M, Buffa E, Simon A, Ashton J, Mcgregor R, Foster DJ. Extrahepatic biliary duct obstruction as a result of involuntary transcavitary implantation of hair in a cat. $\mathrm{J}$ Feline Med Surg [Internet]. 2015 [cited 2020 Jun 4];1(2):1-5. Available from: https://journals.sagepub.com/doi/10.1177/2055116915610359

17. Ribeiro VM. Controle de helmintos de cães e gatos. In: XIII Congresso Brasileiro de Parasitologia Veterinária \& I Simpósio Latino americano de Ricketisioses Ouro Preto, Minas Gerais. Rev Bras Parasitol Vet [Internet]. 2004 [cited 2020 Jun 4];13 Supl 1:8895. Available

from: http://www.lamdosig.ufba.br/Disciplinas/mev160/arquivos/CONTROLE\%20DE\%20HE LMINTOS\%20DE\%20C\%C3\%83ES\%20E\%20GATOS.pdf

18. Braga RR, Teixeira AC, Oliveira JA, Cavalcanti LP. Prevalence of Platynosomum fastosum infection in free roaming cats in northeastern Brazil: fluke burden and grading of lesions. Vet Parasitol [Internet]. 2016 [cited 2020 Jun 4];227(1):20-5. Available from: https://www.sciencedirect.com/science/article/abs/pii/S0304401716302801?via\%3Dihub

19. Carvalho TK, Batista LSO, Sampaio LAL, Aragão AP. Diagnóstico anatomohistopatológico de platinosomose em felino: relato de caso. Acta Biomed Bras [Internet]. 2017 [cited 2020 Jun 4];8(2):140-6. Available from: https://www.actabiomedica.com.br/index.php/acta/article/view/226/179

20. Azevedo FD. Alterações hepatobiliares em gatos domésticos (Felis catus domesticus) parasitados por Platynosomum illiciens (Braun, 1901) Kossak, 1910 observadas através dos exames radiográfico, ultrassonográfico e de tomografia computadorizada [dissertação]. Rio de Janeiro: Universidade Federal Rural do Rio de Janeiro; 2008.

21. Leal PDSA, Campos DP, Rodrigues MLA, Botelho GG, Labarthe NV. Avaliação da administração oral de ácido ursodesoxicólico (AUDC) no diagnóstico da infecção natural por Platynosomum illiciens em gatos. Rev Bras Med Vet [Internet]. 2011 [cited 2020 Jun 6];33(4):229-33. Available from: http://rbmv.org/index.php/BJVM/article/view/815?articlesBySameAuthorPage=2

Ferraz A, Lima CM, Barwaldt ET, Bierhals ES, Chagas BC, et al. Platinossomose em felino doméstico no município de Pelotas, RS, Brasil. Vet. e Zootec. 2021; v28: 001-008. 
22. Sobral MCGO, Sousa SAP, Ribeiro TMP, Galvão SR, Santos RM, Silva RA, et al. Infection by Platynosomum illiciens $(=P$. fastosum) in domestic cats of Araguaína, Tocantins, northern Brazil. Rev Bras Parasitol Vet [Internet]. 2019 [cited 2020 Jun 6];28(4):786-9. Available from: https://www.scielo.br/pdf/rbpv/v28n4/1984-2961-rbpvS1984-29612019070.pdf

23. Zanutto MS, Almeida MAO, Junquilho AB, Silva MAS, Silveira RX, Fatal PL. Uso do Endal Gatos no tratamento da platinossomíase felina. Hora Vet. 2012;185:12-6.

24. Soldan MH, Marques SMT. Platinosomose: abordagem na clínica felina. Rev FZVA. 2011;18(1):46-67.

25. Norsworthy GD. Flukes: liver, biliary and pancreatic. In: Norsworthy GD, Crystal MA, Grace SF, Tilley LP. The feline patient. 4ed. Ames: Blackwell; 2011. Cap.76, p.193-4.

26. Andrade SF, Santarém VA. Endoparasiticidas e ectoparasiticidas. In: Andrade SF. Manual de terapêutica veterinária. 2a ed. São Paulo: Roca; 2008. p.437-76.

27. Willard MD, Fossum TW. Doenças da vesícula biliar e do sistema biliar extra-hepático. In: Ettinger SJ, Feldman EC. Tratado de medicina interna veterinária: doenças do cão e gato. 5a ed. Rio de Janeiro. Guanabara Koogan; 2004. v.2, cap.145, p.1413-7.

Recebido em: 19/11/2020

Aceito em: 15/03/2021

Ferraz A, Lima CM, Barwaldt ET, Bierhals ES, Chagas BC, et al. Platinossomose em felino doméstico no município de Pelotas, RS, Brasil. Vet. e Zootec. 2021; v28: 001-008. 